\title{
Complete Genome Sequence of Actinomadura Parvosata Subsp. Kistnae, A Rich Source of Novel Natural Product (Bio-)Chemistry
}

\author{
Kalina Kusserow and Tobias A. M. Gulder ${ }^{\bowtie}$ \\ Biosystems Chemistry, Department of Chemistry and Center for Integrated Protein Science Munich (CIPSM), Technical University of \\ Munich, Lichtenbergstraße 4, 85748 Garching, Germany. \\ $\triangle$ Corresponding author: E.Mail: tobias.gulder@ch.tum.de \\ (C) Ivyspring International Publisher. This is an open access article distributed under the terms of the Creative Commons Attribution (CC BY-NC) license \\ (https://creativecommons.org/licenses/by-nc/4.0/). See http://ivyspring.com/terms for full terms and conditions.
}

Received: 2017.02.15; Accepted: 2017.05.14; Published: 2017.07.02

\begin{abstract}
The soil dwelling actinomycete strain Actinomadura parvosata subsp. kistnae is the producer of the antiviral antibiotics kistamicin $A$ and $B$. Genome sequencing and bioinformatic analysis revealed the presence of the kistamycin biosynthetic gene cluster responsible for the formation of these non-ribosomal peptides as well as an impressive number of yet uncharacterized biosynthetic pathways. This includes polyketide, ribosomal and non-ribosomal peptide and a large number of terpenoid biosynthetic loci encoding yet unknown natural products. The genomic data of this strain is thus a treasure trove for genome mining for novel functional metabolites and new biocatalysts.
\end{abstract}

Key words: Actinomadura parvosata subsp. kistnae, whole-genome sequencing, Kistamicin.

\section{Introduction}

Actinomycetes are well known to produce a wealth of secondary metabolites with diverse biological activities and complex structures [1]. Included in this broad range of compounds are antibiotics, antivirals and anticancer agents from important natural product classes, such as peptides, polyketides or terpenes [2]. Unfortunately, many more promising secondary metabolites encoded in actinomycete genomes remain undiscovered because their biosynthetic gene clusters are not expressed under standard fermentation conditions $[3,4]$. In recent years, these putatively silent gene clusters have become accessible by genome mining $[5,6]$. In our search for yet undiscovered natural product chemistry and biochemistry we selected the actinomycete strain Actinomadura parvosata subsp. kistnae (strain designation S382-8) as a promising target organism. It was isolated from a soil sample collected near the Kistna River in India and shown to produce the potent antiviral antibiotics kistamicin A and B [7]. These secondary metabolites exhibit potent antiviral activity against influenza virus type A as well as antimicrobial activity against Gram-positive bacteria and putatively belong to the class of non-ribosomal peptides [7]. To get further insights into the kistamicin biosynthesis and to explore the strains whole metabolic potential we sequenced the genome of Actinomadura parvosata subsp. kistnae (Table 1).

In the draft genome sequence we indeed identified the $48 \mathrm{~kb}$ kistamicin biosynthetic gene cluster, showing the typical NRPS assembly line organization $[8,9]$. Furthermore, in silico analysis with the program antiSMASH [8] revealed 33 other gene clusters for the biosynthesis of secondary metabolites. This places Actinomadura parvosata subsp. kistnae 
among those actinomycete strains that harbour the most biosynthetic gene clusters found in this group of bacteria [10]. antiSMASH [8] revealed six ribosomal and non-ribosomal peptide Synthetase (NRPS) systems ranging from $30 \mathrm{~kb}$ to $69 \mathrm{~kb}$ in size as well as six polyketide synthase (PKS) clusters ranging from $34 \mathrm{~kb}$ to $51 \mathrm{~kb}$ (3 type I PKS, 1 type II PKS, 1 type III PKS, 1 unspecified) and two mixed NRPS-PKS (both $>100 \mathrm{~kb}$ ). Additionally, seven terpene biosynthetic gene clusters (21 kb to $22 \mathrm{~kb}$ ), 4 lassopeptide, 3 bacteriocin, 2 lantipeptide, 1 mixed lassopeptidelantipeptide, 2 siderophore and 1 unspecified cluster have been detected by antiSMASH. Only two of these clusters show $100 \%$ similarity to already characterized pathways, namely those encoding geosmin and 2-methylisoborneol. All other biosynthetic loci have low similarity to previously characterized gene clusters. Together with the sheer number of biosynthetic gene clusters these findings underline the high potential of this organism for the discovery of novel chemistry and biochemical transformations useful in biomedicine and biotechnological applications.

Table 1. Genome features of Actinomadura parvosata subsp. kistnae.

\begin{tabular}{ll}
\hline Features & Chromosome \\
\hline Length $(\mathrm{bp})$ & $13,559,781$ \\
G+C content $(\%)$ & $71.7 \%$ \\
CDS & 12784 \\
rRNA genes & 17 \\
tRNA genes & 63 \\
Biosynthetic gene clusters & 34 \\
\hline
\end{tabular}

The genomic sequence of Actinomadura parvosata subsp. kistnae was obtained by PacBio single molecule real-time (SMRT®) sequencing. The raw data was generated from three SMRT cells, resulting in a total of approx. 173.600 reads with an average size of $5.9 \mathrm{~kb}$ and a N50 read length of $10 \mathrm{~kb}$. Assembly of this data by GATC resulted in 36 polished contigs with an average coverage of 61.3. In total this leads to a size of the chromosome of this strain of approx. 13.56 MBp and thus places it among the largest actinobacterial genome sequences known to date [10]. The $G+C$ content is $71.7 \%$. Importantly, approx. $10 \%$ of the very large genome of the strain is devoted to the biosynthesis of specialized metabolites, as shown by antiSMASH analysis [8] described above. The genome was annotated using the RAST server [11] and the software Geneious version 8.1.8 [12], resulting in the annotation of 12784 coding sequences (CDSs) as well as 17 rRNA genes and 34 tRNA loci.

The complete genome sequence was deposited at EMBL under Accession Np. [PRJEB19374].

\section{Acknowledgements}

We thank the German Research Foundation DFG (GU-1233/1-1 and CIPSM) and the Hans-FischerGesellschaft e. V. for generous funding. This work was supported by the German Research Foundation (DFG) and the Technical University of Munich (TUM) in the framework of the Open Access Publishing Program.

\section{Competing Interests}

The authors have declared that no competing interests exist.

\section{References}

1. Nett M., Ikeda H., Moore B.S. Genomic basis for natural product biosynthetic diversity in the actinomycetes. Nat. Prod. Rep. 2009; 26: 1362-1384.

2. Nawani N., Aigle B., Mandal A., Bodas M., Ghorbel S., Prakash D. Actinomycetes: Role in biotechnology and medicine. Biomed. Res. Int. 2013; 2013: 687190.

3. Hertweck C. Hidden biosynthetic treasures brought to light. Nat. Chem. Biol. 2009; 5: 450-452.

4. Devine R., Hutchings M.I., Holmes N.A. Future directions for the discovery of antibiotics from actinomycete bacteria. Emerging Topics in Life Sciences 2017; [Epub ahead of print].

5. Rutledge P.J., Challis G.L. Discovery of microbial natural products by activation of silent biosynthetic gene clusters. Nat. Rev. Micro. 2015; 13: 509-523.

6. Ziemert N., Alanjary M., Weber T. The evolution of genome mining in microbes - a review. Nat. Prod. Rep. 2016; 33: 988-1005.

7. Naruse N., Tenmyo O., Kobaru S., Hatori M., Tomita K., Hamagishi Y., Oki T. New antiviral antibiotics, kistamicins A and B I. Taxonomy, production, isolation, physico-chemical properties and biological activities. J. Antibiot. 1993; 46: 1804-1811.

8. Weber T., Blin K., Duddela S., et al. antiSMASH 3.0 a comprehensive resource for the genome mining of biosynthetic gene clusters. Nucleic Acids Res. 2015; 43: W237-243.

9. Bachmann B.O., Ravel J. Methods for in silico prediction of microbial polyketide and nonribosomal peptide biosynthetic pathways from DNA sequencing data. Methods Enzymol. 2009; 458: 181-217.

10. Doroghazi J.R., Metcalf W.W. Comparative genomics of actinomycetes with a focus on natural product biosynthetic genes. BMC Genomics 2013; 14: 611-623.

11. Aziz R.K, Bartels D., Best A.A., et al. The RAST Server: Rapid Annotations using Subsystems Technology. BMC Genomics 2008; 9: 75-89.

12. Kearse M., Moir R., Wilson A., et al. Geneious Basic: an integrated and extendable desktop software platform for the organization and analysis of sequence data. Bioinformatics. 2012; 28: 1647-1649. 